\title{
Brachytherapie verlängert das Leben
}

Patientinnen mit inoperablem Endometriumkarzinom im Stadium I profitieren von einer Brachytherapie und eventuell auch von einer zusätzlichen externen Strahlentherapie unabhängig vom Alter und Tumorgrad. Sowohl das krankheitsfreie als auch das Gesamtüberleben sind im Vergleich zu lediglich extern bestrahlten Frauen deutlich besser. Das zeigen die Daten einer Registerstudie.

Onkologen der Universität Washington, $\mathrm{MO} / \mathrm{USA}$, werteten retrospektiv die Daten von 18 Registern aus, die im Rahmen des National Cancer Institute's Surveillance, Epidemiology an End Result (SEER)-Programms innerhalb des Zeitraums 1998 bis Ende 2011 angefallen waren. Für die Auswertung standen die Daten von insgesamt 460 Frauen mit einem
Endometriumkarzinom im Stadium I zur Verfügung, die nicht operiert wurden und eine Strahlentherapie erhielten - entweder lediglich eine externe Radiotherapie $(n=260)$ oder eine Brachytherapie mit oder ohne externe Strahlentherapie $(\mathrm{n}=200)$.

Als einzigen Faktor, der signifikant mit dem Einsatz einer Brachytherapie verbunden war, identifizierten die Statistiker ein niedrigeres Patientenalter (median 72 vs. 76 Jahre, $p=0,001$ ). Bei den brachytherapierten Frauen ergaben sich signifikant höhere Raten für das 3-Jahres-Gesamtüberleben (60 vs. $47 \%$; $\mathrm{p}<0,001$ ) sowie das krankheitsspezifische Überleben nach drei Jahren (82 vs. $74 \%$; $=0,032$ ). Diese Überlegenheit der Brachytherapie ggf. in Kombination mit externer Strahlenthera-

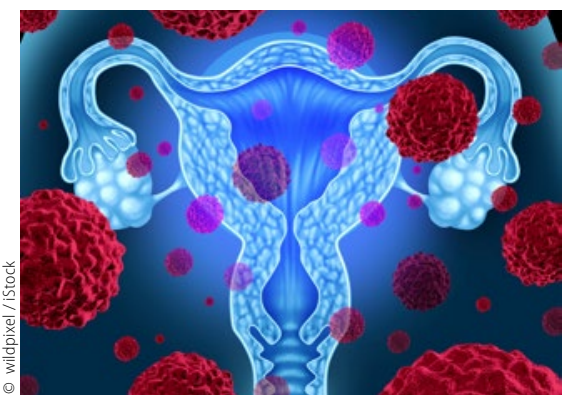

Bei inoperablem Endometriumkarzinom im Stadium I lohnt sich eine Brachytherapie.

pie im Vergleich zu einer lediglich externen Bestrahlung zeigte sich auch in multivariaten sowie alters- und gradinggematchten Analysen. Barbara Kreutzkamp

Acharya S et al. Brachytherapy is associated with improved survival in inoperable stage I endometrial adenocarcinoma: A population-based analysis. Int J Radiat Oncol Biol Phys. 2015 June 15 [Epub ahead of print].

\section{Bestrahlung}

\section{Lymphompatienten optimal versorgen}

Die Bestrahlung bei Patienten mit niedriggradigem follikulärem Lymphom im Frühstadium trägt dazu bei, dass fünf und zehn Jahre nach der Behandlung mehr Patienten noch leben. Internationale Leitlinien empfehlen diese Strategie in dieser Patientengruppe. Auch in der deutschen Leitlinie zum follikulären Lymphom wird im Frühstadium der Erkrankung bei Patienten in allen Altersgruppen eine lokale Bestrahlung (involved field) empfohlen, und zwar in einer Gesamtdosis von mindestens $30 \mathrm{~Gy}$.

In einer retrospektiven Studie aus den USA wurde nun anhand der Daten des National Cancer Data Base untersucht, wie gut die Leitlinien zum follikulären Lymphom zwischen 1998 und 2012 umgesetzt wurden und welchen Nutzen die Patienten von der Bestrahlung hatten. Berücksichtigt wurden in der Studie 35.961 Patienten mit follikulärem Lymphom, von denen $63 \%$ im Stadium I waren.

Dabei wurden mehrere Faktoren identifiziert, die sich positiv auf das Überleben auswirkten. Dazu gehörte außer der Bestrahlung unter anderem eine Kombinationschemotherapie, eine Operation, ein
Alter unter 60 Jahren, das Stadium I der Erkrankung, das Fehlen von B-Symptomen (Fieber, Nachtschweiß, Gewichtsverlust) sowie weibliches Geschlecht.

Auffallend war, dass im Lauf der Jahre immer seltener die Bestrahlung eingesetzt wurde. Lag der Anteil im Jahr 1998 noch bei $37 \%$, so sank er bis zum Jahr 2012 auf $24 \%$. Entsprechend stieg in dieser Zeit der Anteil der Patienten, die nur beobachtet bzw. die nur mit einer Monochemotherapie behandelt wurden.

Das mediane Gesamtüberleben betrug 149 Monate, wenn alle Patienten in die
Berechnung einbezogen wurden. Bei Patienten mit initialer Bestrahlung lag der Wert deutlich höher, nämlich bei 179 Monaten. Das 5- bzw. 10-Jahres-Überleben betrug hier $86 \%$ bzw. $68 \%$. Patienten ohne Bestrahlung schnitten deutlich schlechter ab. Das Gesamtüberleben lag in dieser Gruppe bei 133 Monaten. Nach fünf Jahren lebten noch $74 \%$ von ihnen, nach zehn Jahren noch $54 \%$. Die Bestrahlung war folglich mit einer signifikant um absolut $12 \%$ nach fünf Jahren und um $14 \%$ nach zehn Jahren verbesserten Überlebensrate assoziiert.

Peter Leiner

Vargo JA et al. What is the Optimal Management of Early-Stage Low-Grade Follicular Lymphoma in the Modern Era? Cancer. 2015;121(18):3325-34.

\section{kurz notiert}

\section{Personalisierte Vakzine bei malignem Melanom getestet}

Einige Melanompatienten entwickeln eine T-Zell-Immunität, die sich gegen Aminosäure-Austausche richtet. Durch Missense-Mutationen, die zu einem Aminosäure-Austausch führen, entstehen Neoantigene, die der Körper als fremd erkennt und die somit eine Immunantwort auslösen. Wissenschaftler der Universität Washington, St. Louis, MO/USA, entwickelten aus den Neoantigenen zusammen mit dendritischen Zellen einen Impfstoff. Eine Phase-I-Studie, in der diese Vakzine an drei Patienten mit fortgeschrittenem Melanom getestet wurde, lieferte vielversprechende Ergebnisse. Es zeigte sich, dass diese Impfung die T-Zell-Antwort gegen die Neoantigene verstärkte [Carreno BM et al. Science. 2015;348(6236):803-8].

Judith Neumaier 